Advanced Studies in Theoretical Physics

Vol. 8, 2014, no. 27, 1191 - 1198

HIKARI Ltd, www.m-hikari.com

http://dx.doi.org/10.12988/astp.2014.411151

\title{
Some Identities for the Higher-order $q$-Bernoulli Polynomials of the Second Kind under the Third Dihedral Group $D_{3}$
}

\author{
Taekyun Kim \\ Department of Mathematics \\ Kwangwoon University \\ Seoul 139-701, Republic of Korea \\ Dae San Kim \\ Department of Applied Mathematics \\ Sogang University \\ Seoul 121-742, Republic of Korea \\ Yu Seon Jang \\ Department of Applied Mathematics \\ Kangnam University \\ Youngin 446-702, Republic of Korea \\ Seog-Hoon Rim \\ Department of Mathematics Education \\ Kyungpook National University \\ Taegu 702-701, Republic of Korea
}

Copyright (c) 2014 Taekyun Kim, Dae San Kim, Yu Seon Jang and Seog-Hoon Rim. This is an open access article distributed under the Creative Commons Attribution License, which permits unrestricted use, distribution, and reproduction in any medium, provided the original work is properly cited. 


\begin{abstract}
In this paper, we consider the higher-order $q$-Bernoulli polynomials of the second kind and investigate some symmetric identities under the third Dihedral group $D_{3}$ which are derived from multivariate $p$-adic invariant integral on $\mathbb{Z}_{p}$.
\end{abstract}

Keywords: $p$-adic invariant integral on $\mathbb{Z}_{p}$, higher-order $q$-Bernoulli numbers and polynomials of the second kind

\title{
1. INTRODUCTION
}

Let $p$ be a fixed prime number. Throughout this paper, $\mathbb{Z}_{p}, \mathbb{Q}_{p}$, and $\mathbb{C}_{p}$ will, respectively, denote the ring of $p$-adic rational integers, the field of $p$-adic rational numbers and the completion of the algebraic closure of $\mathbb{Q}_{p}$. Let $v_{p}$ be the normalized exponential valuation of $\mathbb{C}_{p}$ with $|p|_{p}=1 / p$ and let $q$ be an indeterminate in $\mathbb{C}_{p}$ with $|1-q|_{p}<p^{-1 /(p-1)}$. As is well known, the $q$-number of $x$ is defined by $[x]_{q}=\left(1-q^{x}\right) /(1-q)$. Note that $\lim _{q \rightarrow 1}[x]_{q}=x$. Let us assume that $f(x)$ is uniformly differentiable function on $\mathbb{Z}_{p}$. The $p$-adic invariant integral on $\mathbb{Z}_{p}$ is defined to be

$$
\begin{aligned}
\int_{\mathbb{Z}_{p}} f(x) d \mu_{0}(x) & =\lim _{N \rightarrow \infty} \sum_{x=0}^{p^{N}-1} f(x) \mu_{0}\left(x+p^{N} \mathbb{Z}_{p}\right) \\
& =\lim _{N \rightarrow \infty} \frac{1}{p^{N}} \sum_{x=0}^{p^{N}-1} f(x), \quad(\text { see }[8,10,12]) .
\end{aligned}
$$

From (1), we have

$$
\int_{\mathbb{Z}_{p}} f(x+1) d \mu_{0}(x)-\int_{\mathbb{Z}_{p}} f(x) d \mu_{0}(x)=f^{\prime}(0),
$$

where $f^{\prime}(0)=d f(x) /\left.d x\right|_{x=0}$. By (2), we easily get

$$
\int_{\mathbb{Z}_{p}} \exp (t(x+y)) d \mu_{0}(y)=\frac{t}{e^{t}+1} e^{x t}=\sum_{n=0}^{\infty} B_{n}(x) \frac{t^{n}}{n !} .
$$

where $B_{n}(x)$ are called Bernoulli polynomials. When $x=0, B_{n}=B_{n}(0)$ are called the Bernoulli numbers (see $[8,9,10]$ ).

Recently, several authors have studied variously $q$-analogue of Bernoulli numbers and polynomials (see [1-20]). In [8], D. S. Kim and T. Kim have considered $q$-Benoulli polynomials of the second kind as follows:

$$
\int_{\mathbb{Z}_{p}} \exp \left([x+y]_{q} t\right) d \mu_{0}(y)=\sum_{n=0}^{\infty} \beta_{n, q}(x) \frac{t^{n}}{n !} .
$$


When $x=0, \beta_{n, q}=\beta_{n, q}(0)$ are called the $q$-Bernoulli numbers of the second kind. From (4), we can derive the following recurrence relation:

$$
\beta_{0, q}=1,\left(q \beta_{q}+1\right)^{n}-\beta_{n, q}=\left\{\begin{array}{cc}
\frac{\log q}{q-1}, & \text { if } n=1 \\
0, & \text { if } n>1
\end{array}\right.
$$

with the usual convention about replacing $\beta_{q}^{n}$ by $\beta_{n, q}$ (see $\left.[6,7,8]\right)$.

By (4) and (5), we get

$$
\beta_{n, q}(x)=\frac{\log q}{q-1} \frac{1}{(1-q)^{n}} \sum_{k=0}^{n}\left(\begin{array}{l}
n \\
k
\end{array}\right)(-1)^{k} \frac{k}{[k]_{q}} q^{k x}, \text { (see [8]). }
$$

For $r \in \mathbb{N}$, we consider the higher-order $q$-Bernoulli polynomials of the second kind as follows :

$$
\int_{\mathbb{Z}_{p}} \cdots \int_{\mathbb{Z}_{p}} \exp \left(\left[x+\sum_{\ell=1}^{r} y_{\ell}\right]_{q} \times t\right) d \mu_{0}\left(y_{1}\right) \cdots d \mu_{0}\left(y_{r}\right)=\sum_{n=0}^{\infty} \beta_{n, q}^{(r)}(x) \frac{t^{n}}{n !} .
$$

When $x=0, \beta_{n, q}^{(r)}=\beta_{n, q}^{(r)}(0)$ are called the Bernoulli numbers of order $r$. Thus, by (7), we get

$$
\begin{aligned}
\beta_{n, q}^{(r)}(x) & =\int_{\mathbb{Z}_{p}} \cdots \int_{\mathbb{Z}_{p}}\left[x+\sum_{\ell=1}^{r} y_{\ell}\right]_{q}^{n} d \mu_{0}\left(y_{1}\right) \cdots \mu_{0}\left(y_{r}\right) \\
& =\left(\frac{\log q}{q-1}\right)^{r} \frac{1}{(1-q)^{n}} \sum_{k=0}^{n}\left(\begin{array}{l}
n \\
k
\end{array}\right)(-1)^{k}\left(\frac{k}{[k]_{q}}\right)^{r} q^{k x} .
\end{aligned}
$$

In this paper, we investigate some symmetric identities for the higher-order $q$-Bernoulli polynomials of the second kind under the third Dihedral group $D_{3}$.

\section{Some IDEntities FOR THE HigheR-ORDER $q$-BeRnoulli} POLYNOMIALS OF THE SECOND KIND

For $w_{1}, w_{2}, w_{3} \in \mathbb{Z}$ with $w_{1} \geq 1, w_{2} \geq 1, w_{3} \geq 1$, let

$$
f_{1}\left(y_{1}, \cdots, y_{r}\right)=\exp \left\{\left[w_{1} w_{2} w_{3} x+\sum_{\ell=1}^{r}\left(w_{2} w_{3} y_{\ell}+w_{1} w_{3} i_{\ell}+w_{1} w_{2} j_{\ell}\right)\right]_{q} \times t\right\}
$$

and

$$
g_{1}\left(y_{1}, \cdots, y_{r}\right)=\exp \left\{\left[w_{1} w_{2} w_{3} x+\sum_{\ell=1}^{r}\left(w_{2} w_{3}\left(h_{\ell}+w_{1} y_{\ell}\right)+w_{1} w_{3} i_{\ell}+w_{1} w_{2} j_{\ell}\right)\right]_{q} \times t\right\} .
$$


Then we see that

$$
\begin{aligned}
& \int_{\mathbb{Z}_{p}} \cdots \int_{\mathbb{Z}_{p}} f_{1}\left(y_{1}, \cdots, y_{r}\right) d \mu_{0}\left(y_{1}\right) \cdots d \mu_{0}\left(y_{r}\right) \\
= & \lim _{N \rightarrow \infty}\left(\frac{1}{p^{N}}\right)^{r} \sum_{y_{1}, \cdots, y_{r}=0}^{p^{r}-1} f_{1}\left(y_{1}, \cdots, y_{r}\right) \\
= & \lim _{N \rightarrow \infty}\left(\frac{1}{w_{1} p^{N}}\right)^{r} \sum_{h_{1}, \cdots, h_{r}=0}^{w_{1}-1} \sum_{y_{1}, \cdots, y_{r}=0}^{p^{N}-1} g_{1}\left(y_{1}, \cdots, y_{r}\right),
\end{aligned}
$$

From (9), we can derive the following equation:

$$
\begin{aligned}
& \frac{1}{\left(w_{2} w_{3}\right)^{r}} \sum_{i_{1}, \cdots, i_{r}=0}^{w_{2}-1} \sum_{j_{1}, \cdots, j_{r}=0}^{w_{3}-1} \int_{\mathbb{Z}_{p}} \cdots \int_{\mathbb{Z}_{p}} f_{1}\left(y_{1}, \cdots, y_{r}\right) d \mu_{0}\left(y_{1}\right) \cdots d \mu_{0}\left(y_{r}\right) \\
= & \lim _{N \rightarrow \infty}\left(\frac{1}{w_{1} w_{2} w_{3} p^{N}}\right)^{r} \sum_{i_{1}, \cdots, i_{r}=0}^{w_{2}-1} \sum_{j_{1}, \cdots, j_{r}=0}^{w_{3}-1} \sum_{h_{1}, \cdots, h_{r}=0}^{w_{1}-1} \sum_{y_{1}, \cdots, y_{r}=0}^{p^{N}-1} g_{1}\left(y_{1}, \cdots, y_{r}\right) .
\end{aligned}
$$

By the same method as (10), we get

$$
\begin{aligned}
& \frac{1}{\left(w_{1} w_{3}\right)^{r}} \sum_{i_{1}, \cdots, i_{r}=0}^{w_{3}-1} \sum_{j_{1}, \cdots, j_{r}=0}^{w_{1}-1} \int_{\mathbb{Z}_{p}} \cdots \int_{\mathbb{Z}_{p}} f_{2}\left(y_{1}, \cdots, y_{r}\right) d \mu_{0}\left(y_{1}\right) \cdots d \mu_{0}\left(y_{r}\right) \\
= & \lim _{N \rightarrow \infty}\left(\frac{1}{w_{1} w_{2} w_{3} p^{N}}\right)^{r} \sum_{i_{1}, \cdots, i_{r}=0}^{w_{3}-1} \sum_{j_{1}, \cdots, j_{r}=0}^{w_{1}-1} \sum_{h_{1}, \cdots, h_{r}=0}^{w_{2}-1} \sum_{y_{1}, \cdots, y_{r}=0}^{p^{N}-1} g_{2}\left(y_{1}, \cdots, y_{r}\right) .
\end{aligned}
$$

where

$$
f_{2}\left(y_{1}, \cdots, y_{r}\right)=\exp \left\{\left[w_{1} w_{2} w_{3} x+\sum_{\ell=1}^{r}\left(w_{3} w_{1} y_{\ell}+w_{2} w_{1} i_{\ell}+w_{2} w_{3} j_{\ell}\right)\right]_{q} \times t\right\}
$$

and

$g_{2}\left(y_{1}, \cdots, y_{r}\right)=\exp \left\{\left[w_{1} w_{2} w_{3} x+\sum_{\ell=1}^{r}\left(w_{3} w_{1}\left(h_{\ell}+w_{2} y_{\ell}\right)+w_{2} w_{1} i_{\ell}+w_{2} w_{3} j_{\ell}\right)\right]_{q} \times t\right\}$.

Therefore, by (10) and (11), we obtain the following theorem.

Theorem 2.1. Let $w_{1}, w_{2}, w_{3} \in \mathbb{Z}$ with $w_{1} \geq 1, w_{2} \geq 1, w_{3} \geq 1$. Then the following expressions

$$
\frac{1}{\left(w_{\sigma(2)} w_{\sigma(3)}\right)^{r}} \sum_{i_{1}, \cdots, i_{r}=0}^{w_{\sigma(2)}-1} \sum_{j_{1}, \cdots, j_{r}=0}^{w_{\sigma(3)}-1} \int_{\mathbb{Z}_{p}} \cdots \int_{\mathbb{Z}_{p}} f\left(y_{1}, \cdots, y_{r}\right) d \mu_{0}\left(y_{1}\right) \cdots d \mu_{0}\left(y_{r}\right)
$$


are the same for any $\sigma \in D_{3}$, where

$$
\begin{aligned}
& f\left(y_{1}, \cdots, y_{r}\right)= \\
& \exp \left\{\left[w_{\sigma(1)} w_{\sigma(2)} w_{\sigma(3)} x+\sum_{\ell=1}^{r}\left(w_{\sigma(2)} w_{\sigma(3)} y_{\ell}+w_{\sigma(1)} w_{\sigma(3)} i_{\ell}+w_{\sigma(1)} w_{\sigma(2)} j_{\ell}\right)\right]_{q} \times t\right\} .
\end{aligned}
$$

By (8), we get

$$
\begin{aligned}
& \int_{\mathbb{Z}_{p}} \cdots \int_{\mathbb{Z}_{p}} \exp \left\{\left[w_{1} w_{2} w_{3} x+\sum_{\ell=1}^{r}\left(w_{2} w_{3} y_{\ell}+w_{1} w_{3} i_{\ell}+w_{1} w_{2} j_{\ell}\right)\right]_{q} \times t\right\} d \mu_{0}\left(y_{1}\right) \cdots d \mu_{0}\left(y_{r}\right) \\
= & \sum_{n=0}^{\infty}\left[w_{2} w_{3}\right]_{q}^{n} \int_{\mathbb{Z}_{p}} \cdots \int_{\mathbb{Z}_{p}}\left[w_{1} x+\sum_{\ell=1}^{r}\left(y_{\ell}+\frac{w_{1}}{w_{2}} i_{\ell}+\frac{w_{1}}{w_{3}} j_{\ell}\right)\right]_{q^{w_{2} w_{3}}}^{n} d \mu_{0}\left(y_{1}\right) \cdots d \mu_{0}\left(y_{r}\right) \frac{t^{n}}{n !} \\
= & \sum_{n=0}^{\infty}\left[w_{2} w_{3}\right]_{q}^{n} \beta_{n, q^{w_{2} w_{3}}}^{(r)}\left(w_{1} x+\sum_{\ell=1}^{r}\left(\frac{w_{1}}{w_{2}} i_{\ell}+\frac{w_{1}}{w_{3}} j_{\ell}\right)\right) .
\end{aligned}
$$

Therefore, by Theorem 1 and (12), we obtain the following theorem.

Theorem 2.2. Let $w_{1}, w_{2}, w_{3} \in \mathbb{Z}$ with $w_{1} \geq 1, w_{2} \geq 1, w_{3} \geq 1$. For any non-negative integer $n$, the following expressions

$$
\frac{\left[w_{\sigma(2)} w_{\sigma(3)}\right]_{q}^{n}}{\left(w_{\sigma(2)} w_{\sigma(3)}\right)^{r}} \sum_{i_{1}, \cdots, i_{r}=0}^{w_{\sigma(2)}-1} \sum_{i_{1}, \cdots, i_{r}=0}^{w_{\sigma(3)}-1} \beta_{n, q^{w_{\sigma(2)}} w_{\sigma(3)}}^{(r)}\left(w_{\sigma(1)} x+\sum_{\ell=1}^{r}\left(\frac{w_{\sigma(1)}}{w_{\sigma(2)}} i_{\ell}+\frac{w_{\sigma(1)}}{w_{\sigma(3)}} j_{\ell}\right)\right)
$$

are the same for any $\sigma \in D_{3}$.

Now, we observe that

$$
\begin{aligned}
& {\left[w_{1} x+\sum_{\ell=1}^{r}\left(y_{\ell}+\frac{w_{1}}{w_{2}} i_{\ell}+\frac{w_{1}}{w_{3}} j_{\ell}\right)\right]_{q^{w_{2} w_{3}}} } \\
= & \frac{1-q^{\sum_{\ell=1}^{r}\left(w_{1} w_{3} i_{\ell}+w_{1} w_{2} j_{\ell}\right)}}{1-q^{w_{2} w_{3}}}+q^{\sum_{\ell=1}^{r}\left(w_{1} w_{3} i_{\ell}+w_{1} w_{2} j_{\ell}\right)}\left[\sum_{\ell=1}^{r} y_{\ell}+w_{1} x\right]_{q^{w_{2} w_{3}}} \\
= & \frac{\left[w_{1}\right]_{q}}{\left[w_{2} w_{3}\right]_{q}}\left[\sum_{\ell=1}^{r}\left(w_{3} i_{\ell}+w_{2} j_{\ell}\right)\right]_{q^{w_{1}}}+q^{\sum_{\ell=1}^{r}\left(w_{1} w_{3} i_{\ell}+w_{1} w_{2} j_{\ell}\right)}\left[\sum_{\ell=1}^{r} y_{\ell}+w_{1} x\right]_{q^{w_{2} w_{3}}} .
\end{aligned}
$$


From (13), we can derive the following equation :

$$
\begin{aligned}
& \int_{\mathbb{Z}_{p}} \cdots \int_{\mathbb{Z}_{p}}\left[w_{1} x+\sum_{\ell=1}^{r}\left(y_{\ell}+\frac{w_{1}}{w_{2}} i_{\ell}+\frac{w_{1}}{w_{3}} j_{\ell}\right)\right]_{q^{w_{2} w_{3}}}^{n} d \mu_{0}\left(y_{1}\right) \cdots d \mu_{0}\left(y_{r}\right) \\
= & \sum_{k=0}^{n}\left(\begin{array}{l}
n \\
k
\end{array}\right)\left(\frac{\left[w_{1}\right]_{q}}{\left[w_{2} w_{3}\right]_{q}}\right)^{n-k}\left[\sum_{\ell=1}^{r}\left(w_{3} i_{\ell}+w_{2} j_{\ell}\right)\right]_{q^{w_{1}}}^{n-k} q^{k \sum_{\ell=1}^{r}\left(w_{1} w_{3} i_{\ell}+w_{1} w_{2} j_{\ell}\right)} \\
& \times \int_{\mathbb{Z}_{p}} \cdots \int_{\mathbb{Z}_{p}}\left[\sum_{\ell=1}^{r} y_{\ell}+w_{1} x\right]_{q^{w_{2} w_{3}}}^{k} d \mu_{0}\left(y_{1}\right) \cdots d \mu_{0}\left(y_{r}\right) \\
= & \sum_{k=0}^{n}\left(\begin{array}{l}
n \\
k
\end{array}\right)\left(\frac{\left[w_{1}\right]_{q}}{\left[w_{2} w_{3}\right]_{q}}\right)^{n-k}\left[\sum_{\ell=1}^{r}\left(w_{3} i_{\ell}+w_{2} j_{\ell}\right)\right]_{q^{w_{1}}}^{n-k} \\
& \times q^{k \sum_{\ell=1}^{r}\left(w_{1} w_{3} i_{\ell}+w_{1} w_{2} j_{\ell}\right)} \beta_{k, q^{w_{2} w_{3}}}^{(r)}\left(w_{1} x\right)
\end{aligned}
$$

By (10), (12) and (14), we get

$$
\begin{aligned}
& \frac{\left[w_{2} w_{3}\right]_{q}^{n}}{\left(w_{2} w_{3}\right)^{r}} \sum_{i_{1}, \cdots, i_{r}=0}^{w_{2}-1} \sum_{j_{1}, \cdots, j_{r}=0}^{w_{3}-1} \int_{\mathbb{Z}_{p}} \cdots \int_{\mathbb{Z}_{p}} \\
\times & {\left[w_{1} x+\sum_{\ell=1}^{r}\left(y_{\ell}+\frac{w_{1}}{w_{2}} i_{\ell}+\frac{w_{1}}{w_{3}} j_{\ell}\right)\right]_{q^{w_{2} w_{3}}}^{n} d \mu_{0}\left(y_{1}\right) \cdots d \mu_{0}\left(y_{r}\right) } \\
= & \sum_{k=0}^{n}\left(\begin{array}{l}
n \\
k
\end{array}\right) \frac{\left[w_{2} w_{3}\right]_{q}^{k}}{\left(w_{2} w_{3}\right)^{r}}\left[w_{1}\right]_{q}^{n-k} \beta_{k, q^{w_{2} w_{3}}}^{(r)}\left(w_{1} x\right) \sum_{i_{1}, \cdots, i_{r}=0}^{w_{2}-1} \sum_{j_{1}, \cdots, j_{r}=0}^{w_{3}-1} \\
& \times q^{k \sum_{\ell=1}^{r}\left(w_{1} w_{3} i_{\ell}+w_{1} w_{2} j_{\ell}\right)}\left[\sum_{\ell=1}^{r}\left(w_{3} i_{\ell}+w_{2} j_{\ell}\right)\right]_{q^{w_{1}}}^{n-k} \\
= & \sum_{k=0}^{n}\left(\begin{array}{l}
n \\
k
\end{array}\right) \frac{\left[w_{2} w_{3}\right]_{q}^{k}}{\left(w_{2} w_{3}\right)^{r}}\left[w_{1}\right]_{q}^{n-k} \beta_{k, q^{w_{2} w_{3}}}^{(r)}\left(w_{1} x\right) T_{n, q^{w_{1}}}^{(r)}\left(w_{2}, w_{3} \mid k\right),
\end{aligned}
$$

where

$$
T_{n, q}^{(r)}\left(w_{1}, w_{2} \mid k\right)=\sum_{i_{1}, \cdots, i_{r}=0}^{w_{1}-1} \sum_{j_{1}, \cdots, j_{r}=0}^{w_{2}-1} q^{k \sum_{\ell=1}^{r}\left(w_{2} i_{\ell}+w_{1} j_{\ell}\right)}\left[\sum_{\ell=1}^{r}\left(w_{2} i_{\ell}+w_{1} j_{\ell}\right)\right]_{q}^{n-k}
$$

As this expression is an invariant under third Dihedral group $D_{3}$, we have the following theorem. 
Theorem 2.3. Let $w_{1}, w_{2}, w_{3}$ be any positive integers. Then, for any nonnegative integer $n$, the following expressions

$$
\sum_{k=0}^{n}\left(\begin{array}{l}
n \\
k
\end{array}\right) \frac{\left[w_{\sigma(2)} w_{\sigma(3)}\right]_{q}^{k}}{\left(w_{\sigma(2)} w_{\sigma(3)}\right)^{r}}\left[w_{\sigma(1)}\right]_{q}^{n-k} \beta_{k, q^{(r)} w_{\sigma(2)} w_{\sigma(3)}}\left(w_{\sigma(1)} x\right) T_{n, q^{\prime} w^{(1)}}^{(r)}\left(w_{\sigma(2)}, w_{\sigma(3)} \mid k\right)
$$

are all the same for any $\sigma \in D_{3}$.

\section{REFERENCES}

[1] M. Acikgoz, D. Erdal, and S. Araci, A new approach to $q$-Bernoulli numbers and $q$ Bernoulli polynomials related to $q$-Bernstein polynomials, Adv. Difference Equ. 2010, Art. ID 951764, 9pp. http://dx.doi.org/10.1186/1687-1847-2010-951764

[2] A. Bayad and T. Kim, identities involving values of Bernstein, $q$-Bernoullim and $q$-Euler polynomials, Russ. J. Math. Phys. 18 (2011), no. 2, 133-143. http://dx.doi.org/10.1134/s1061920811020014

[3] M. Can, M. Cenkci, V. Kurt, Y. Simsek, Twisted Dedekind type sums associated with Barnes' type multiple Frobenius-Euler l-functions, Adv. Stud. Contemp. Math. 18 (2009), no. 2, 135-160.

[4] D. Ding, V. Kurt, S. H. Rim, and Y. Simsek, Some identities related to the ApostolEuler and Apostol-Bernoulli polynomials, Adv. Stud. Contemp. Math. 20 (2010), no. 1, $7-21$.

[5] A. S. Hegazi and M. Mansour, A note on $q$-Bernoulli numbers and polynomials, J. Nonlinear Math. Phys. 13 (2006), no. 1. 9-18. http://dx.doi.org/10.2991/jnmp.2006.13.1.2

[6] Y. He, Symmetric identities for Carlitz's $q$-Bernoulli numbers and polynomials, Adv. Difference Equ. 2013, 2013:246, 10pp. http://dx.doi.org/10.1186/1687-1847-2013-246

[7] K.-W. Hwang, D. V. Dolgy, D. S. Kim, T. Kim, S. H. Lee, Some theorems on Bernoulli and Euler numbers, Ars Combin. 109 (2013), 285-297.

[8] D. S. Kim and T. Kim, Symmetry identities of $q$-Bernoulli polynomials of the second kind, Indian J. Pure Appl. Math. (2014), to appear.

[9] D. S. Kim, N. Lee, J. Na, and K. H. Park, Abundant symmetry for higher-order Bernoulli polynomials (1), Adv. Stud. Contemp. Math. 23 (2013), no. 3, 461-482.

[10] T. Kim, $q$-Bernoulli numbers and polynomials associated with Gaussian binomial coefficients, Russ. J. Math. Phys. 15 (2008), no. 1, 51-57. http://dx.doi.org/10.1134/s1061920808010068

[11] T. Kim, C. S. Ryoo, and H. Yi, A note on $q$-Bernoulli numbers and $q$-Bernstein polynomials, Ars Combin. 104 (2012), 437-447.

[12] T. Kim, q-Volkenborn integration, Russ. J. Math. Phys. 9 (2002), no. 3, 288-299.

[13] T. Kim, Sums of products of $q$-Bernoulli numbers, Arch. Math. (Basel) 76 (2001), no. 3, 190-195. http://dx.doi.org/10.1007/s000130050559

[14] Y. H. Kim and K. W. Hwang, Symmetry of power sum and twisted Bernoulli polynomials, Adv. Stud. Contemp. Math. 18 (2009), no. 2, 127-133.

[15] H. Ozden, I. N. Cangul, and Y. Simsek, Remarks on $q$-Bernoulli numbers associated with Daehee numbers, Adv. Stud. Contemp. Math. 18 (2009), no. 1, 41-48.

[16] J. W. Park. New approach to $q$-Bernoulli polynomials with weight or weak weight, Adv. Stud. Contemp. Math. 24 (2014), no. 1, 39-44.

[17] C. S. Ryoo, A note on the weighted q-Euler numbers and polynomials, Adv. Stud. Contemp. Math. 21(2011), 47-54. 
[18] Y. Simsek, Generating functions of the twisted Bernoulli numbers and pplynomials associated with their interpolation functions, Adv. Stud. Contemp. Math. 16 (2008), no. 2, 251-278.

[19] J. J. Seo, S. H. Rim, S. H. Lee, D. V. Dolgy, and T. Kim, $q$-Bernoulli numbers and polynomials related to $p$-adic invariant integral on $\mathbb{Z}_{p}$, Proc. Jangjeon Math. Soc. 16 (2013), no. 3, 321-326.

[20] S. L. Yang and Z. J. Qiao, Some symmetry identities for the Euler polynomial, J. Math. Res. Exposition 30 (2010), no. 3, 457-464.

\section{Received: December 1, 2014; Published: December 22, 2014}

